\title{
Collagen Type I and II Blend Hydrogel with Autologous Mesenchymal Stem Cells as a Scaffold for Articular Cartilage Defect Repair
}

\author{
AUTHORS \\ Claire E. Kilmer ${ }^{1}$, Carly M. Battistoni ${ }^{1}$, Abigail Cox ${ }^{2}$, Gert J. Breur ${ }^{3}$, Alyssa Panitch ${ }^{4,5}$, Julie C. Liu ${ }^{1,4, *}$ \\ AFFILIATIONS \\ ${ }^{1}$ Davidson School of Chemical Engineering, Purdue University, West Lafayette, IN, 47907, USA \\ ${ }^{2}$ Department of Comparative Pathobiology, Purdue University, West Lafayette, IN, 47907, USA \\ ${ }^{3}$ Department of Veterinary Clinical Sciences, Purdue University, West Lafayette, IN, 47907, USA \\ ${ }^{4}$ Weldon School of Biomedical Engineering, Purdue University, West Lafayette, IN, 47907, USA \\ ${ }^{5}$ School of Biomedical Engineering, University of California Davis, Davis, CA, 95616, USA \\ * Corresponding author.
}

\section{SUPPPORTING INFORMATION}

\section{MATERIALS AND METHODS}

Cell Distribution and Viability in Col I/II Hydrogels. The hydrogels were grown in a maintenance medium of low-glucose DMEM supplemented with 10\% fetal bovine serum and 1\% penicillin-streptomycin for 3 days in the inner well of a $\mu$-Slide Angiogenesis chambered coverslip (Ibidi, Munich, Germany). Calcein AM stained live cells green, and ethidium homodimer stained dead cells red. The hydrogels were imaged utilizing a confocal microscope and a 25x objective. Cells were counted utilizing the analyze particles tool in ImageJ (National Institutes of Health, Bethesda, MD).

Contraction. The degree of contraction over time was assessed by measuring the projected crosssectional area of the hydrogel-cell constructs (cell density of $5 \times 10^{6}$ cells/mL) from images at various time points. The cross-sectional area was measured using ImageJ software (National Institutes of Health, Bethesda, MD) and presented as the percentage of original area.

Macroscopic Scoring. After necropsy, the knees of the rabbits were dissected and photographed for macroscopic scoring. The images of the defects in the trochlear groove and the medial condyle were scrambled so that scorers would be blinded to the treatments. The images were scored using 
categories adapted from Van den Borne et al. ${ }^{1}$ In addition, the scores were totaled to give an overall macroscopic score. Three blinded observers scored the defects where $n=8$ defects were scored for the Col I/II and Col I defect and n=16 defects were scored for the empty defects. Kruskal-Wallis one-way ANOVAs and Dunn's multiple comparisons tests revealed that there were no statistical differences between the three treatments investigated in any of the macroscopic scoring categories (degree of defect repair, integration to border zone, and macroscopic appearance) for defects either in the trochlear groove or medial condyle $(\mathrm{p}<0.05)$. There were no statistical differences between the three treatments investigated when comparing overall repair assessment value for defects either in the trochlear groove or medial condyle.

Mechanically Matched Hydrogel Preparation. The amount of collagen incorporated into the gels was determined using a previously described method. ${ }^{2}$ Briefly, the sample was heated at $37^{\circ} \mathrm{C}$ overnight to ensure that the solutions had polymerized. The gel was centrifuged for 15 minutes at $11000 \mathrm{~g}$. The supernatant was separated from the pellet by decanting. The supernatant from each sample was tested $(n=3)$ for the total amount of collagen present using a bicinchoninic acid (BCA) assay (Pierce, Rockford, IL) following the manufacturer's protocol. The amount of protein in the gel was calculated indirectly as the total amount of collagen prepared minus the amount of collagen in the supernatant.

Rheology. Samples of $150 \mu \mathrm{L}$ were polymerized on Tekdon slides at $37^{\circ} \mathrm{C}$ for 12 hours in a humid incubator. Rheological analysis was done using an ARG2 rheometer (TA instruments, New Castle, DE) with a $20 \mathrm{~mm}$ cone geometry. Frequency sweeps were performed at $37^{\circ} \mathrm{C}$ from 0.01 to $1 \mathrm{~Hz}$ with a controlled stress of $0.5 \mathrm{~Pa}$.

Collagen Type II Immunohistological Staining. Immunohistochemistry was performed with an antibody labeling type II collagen (Abcam; clone 2B1.5, ab185430, $0.2 \mathrm{mg} / \mathrm{mL}$ ). Slides were deparaffinized in xylene and rehydrated to water through graded ethanol washes. The rest of the staining protocol was carried out in a Dako Autostainer (Agilent, Santa Clara, CA) at room temperature with Tris buffered saline/Tween washes. Slides were incubated in pepsin (Thermo Fisher Scientific, Waltham, MA) for 15 minutes, and endogenous peroxidase was quenched with $3 \%$ hydrogen peroxide in water for 5 minutes. The samples were blocked for 20 minutes with 2.5\% normal horse serum. Col II mouse monoclonal antibody at a dilution of 1:100 was applied for 60 
minutes. A horse anti-mouse secondary (Vector Labs, Burlingame, CA) was applied for 30 minutes, and ImmPACT DAB (3,3'-diaminobenzidine) horseradish peroxidase (HRP) substrate (Vector Labs, Burlingame, CA) was applied for 5 minutes. Slides were then put in an XL Autostainer (Leica, Buffalo Grove, IL) for hematoxylin counterstaining and addition of a coverslip.

Picrosirius Red Staining and Imaging. Picrosirius red was used to stain tissue, and sections were imaged using polarized light microscopy to examine collagen fibril organization. Cartilage architecture was analyzed using the addition of a polarizer and analyzer to a light microscope to detect changes in the direction of polarized light, or birefringence. 
Table 1S. Primer sequences utilized for quantitative reverse transcription-polymerase chain reaction.

\begin{tabular}{|c|c|c|c|c|c|c|}
\hline $\begin{array}{l}\text { Gene of } \\
\text { Interest }\end{array}$ & $\begin{array}{c}\text { Accession } \\
\text { number }\end{array}$ & & $5^{\prime} \rightarrow 3^{\prime}$ sequence & $\begin{array}{l}\text { Product } \\
\text { length } \\
\text { (bp) }\end{array}$ & $\begin{array}{l}\text { Primer } \\
\text { efficiency }\end{array}$ & Reference \\
\hline \multirow[t]{2}{*}{$\begin{array}{l}\text { Collagen } \\
\text { Type I }\end{array}$} & $\begin{array}{c}\text { NM } \\
001195668.1\end{array}$ & Forward & ATGGATGAGGAAACTGGCAACT & 114 & $101.9 \%$ & Liao, $2010^{3}$ \\
\hline & & Reverse & GCCATCGACAAGAACAGTGTAAGT & & & \\
\hline \multirow{2}{*}{$\begin{array}{l}\text { Collagen } \\
\text { Type II }\end{array}$} & $\begin{array}{c}\text { NM } \\
001195671.1\end{array}$ & Forward & GCCACCGTGCCCAAGAAGAACT & 160 & $103.1 \%$ & Yuan, $2016^{4}$ \\
\hline & & Reverse & ACAGCAGGCGCAGGAAGGTCAT & & & \\
\hline \multirow[t]{2}{*}{$\begin{array}{l}\text { Collagen } \\
\text { Type X }\end{array}$} & $\begin{array}{c}\text { XM } \\
002714724.3\end{array}$ & Forward & GCCAGGACCTCCAGGACTATCA & 103 & $100.0 \%$ & Zheng, $2009^{5}$ \\
\hline & & Reverse & CCСАATGTCTCCTTTCGGTCCA & & & \\
\hline \multirow[t]{2}{*}{ Aggrecan } & $\begin{array}{c}\text { XM } \\
008251723.2\end{array}$ & Forward & CCTACCAGGACAAGGTCTCG & 163 & $98.1 \%$ & Chen, $2016^{6}$ \\
\hline & & Reverse & АСАССТТТСАССАССАССТС & & & \\
\hline \multirow[t]{2}{*}{ Sox9 } & $\begin{array}{c}\text { XM } \\
008271763.2\end{array}$ & Forward & GGAAGCTCTGGAGACTGCTG & 135 & $96.8 \%$ & Zhang, $2012^{7}$ \\
\hline & & Reverse & CGTTCTTCACCGACTTCCTC & & & \\
\hline \multirow[t]{2}{*}{ GAPDH } & $\begin{array}{c}\text { NM } \\
001082253.1\end{array}$ & Forward & CGCCTGGAGAAAGCTGCTA & 104 & $96.0 \%$ & Morigele, $2013^{8}$ \\
\hline & & Reverse & ACGACCTGGTCCTCGGTGTA & & & \\
\hline
\end{tabular}


Table 2S. Histological and histochemical grading scale adapted from O’Driscoll et al ${ }^{9}$.

\begin{tabular}{|c|c|}
\hline & Score \\
\hline \multicolumn{2}{|l|}{ Nature of the predominant tissue } \\
\hline \multicolumn{2}{|l|}{ Cellular morphology } \\
\hline Hyaline articular cartilage & 4 \\
\hline Incompletely differentiated mesenchyme & 2 \\
\hline Fibrous tissue or bone & 0 \\
\hline \multicolumn{2}{|l|}{ Safranin-O staining of the matrix } \\
\hline Normal or nearly normal & 3 \\
\hline Moderate & 2 \\
\hline Slight & 1 \\
\hline None & 0 \\
\hline \multicolumn{2}{|l|}{ Structural characteristics } \\
\hline \multicolumn{2}{|l|}{ Surface regularity } \\
\hline Superficial horizontal lamination & 2 \\
\hline Fissures - 0 to 25 percent of the thickness & 1.5 \\
\hline Fissures - 25 to 100 percent of the thickness & 1 \\
\hline Severe disruption, including fibrillation & 0 \\
\hline \multicolumn{2}{|l|}{ Structural integrity } \\
\hline Normal & 2 \\
\hline Slight disruptions, including cysts & 1 \\
\hline Severe disintegration & 0 \\
\hline \multicolumn{2}{|l|}{ Thickness } \\
\hline 100 percent of normal adjacent cartilage & 2 \\
\hline 50 - 100 percent of normal adjacent cartilage & 1 \\
\hline 0 - 50 percent of normal adjacent cartilage & 0 \\
\hline \multicolumn{2}{|l|}{ Bonding to the Adjacent Cartilage } \\
\hline Bonded at both ends of graft & 2 \\
\hline Bonded at one end and partially at one end & 1.5 \\
\hline Bonded at one end or partially at both ends & 1 \\
\hline Bonded partially at one end & 0.5 \\
\hline Not bonded & 0 \\
\hline \multicolumn{2}{|l|}{ Freedom from cellular changes of degeneration } \\
\hline \multicolumn{2}{|l|}{ Hypocellularity } \\
\hline Normal cellularity & 3 \\
\hline Slight hypocellularity & 2 \\
\hline Moderate hypocellularity & 1 \\
\hline Severe hypocellularity & 0 \\
\hline \multicolumn{2}{|l|}{ Chondrocyte clustering } \\
\hline No clusters & 2 \\
\hline$<25$ percent of the cells & 1 \\
\hline 25 - 100 percent of the cells & 0 \\
\hline \multicolumn{2}{|l|}{ Freedom from degenerative changes in adjacent cartilage } \\
\hline Normal cellularity, no clusters, normal staining & 3 \\
\hline Normal cellularity, mild clusters, moderate staining & 2 \\
\hline Mild or moderate hypocellularity, slight staining & 1 \\
\hline Severe hypocellularity, poor or no staining & 0 \\
\hline
\end{tabular}


A. Maximum Intensity Projection
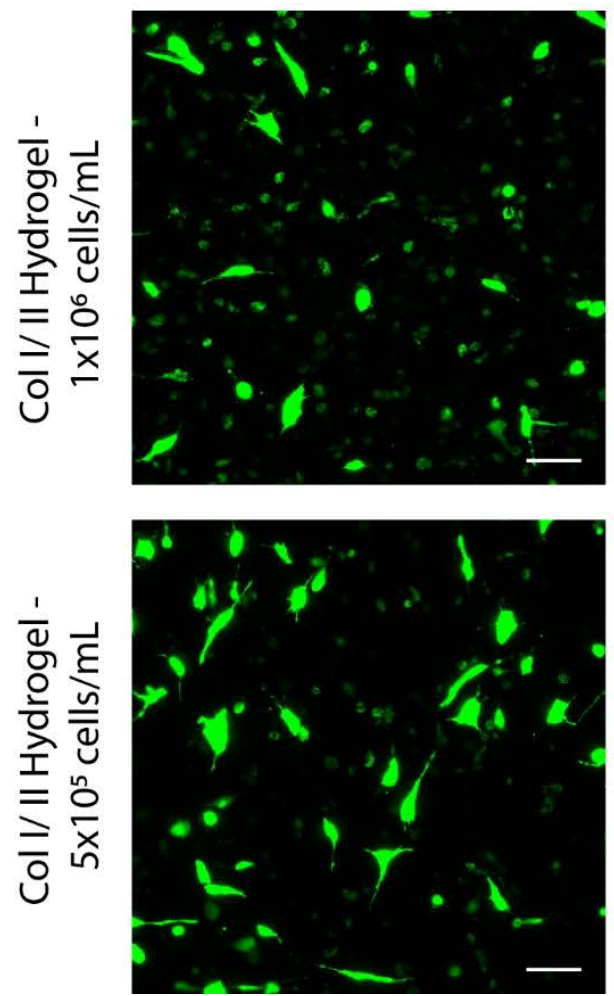

B. Bottom View
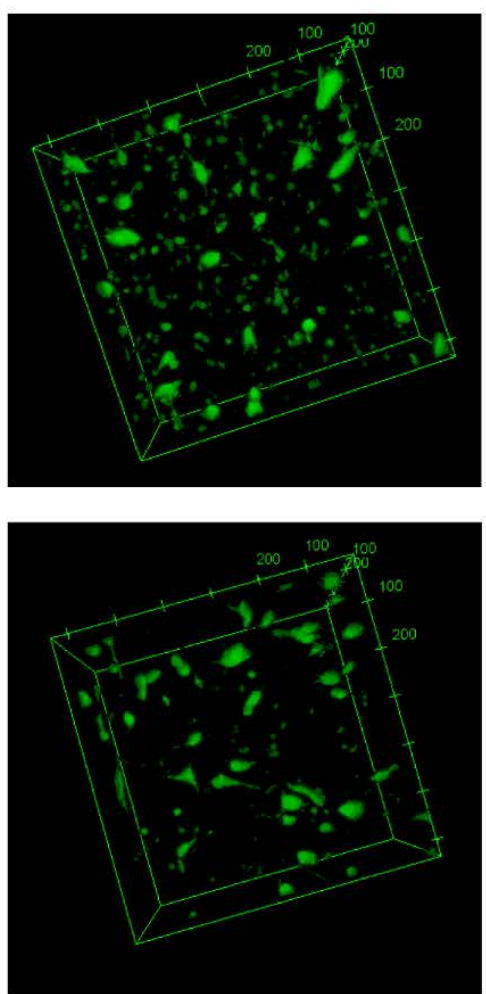

C. Side View
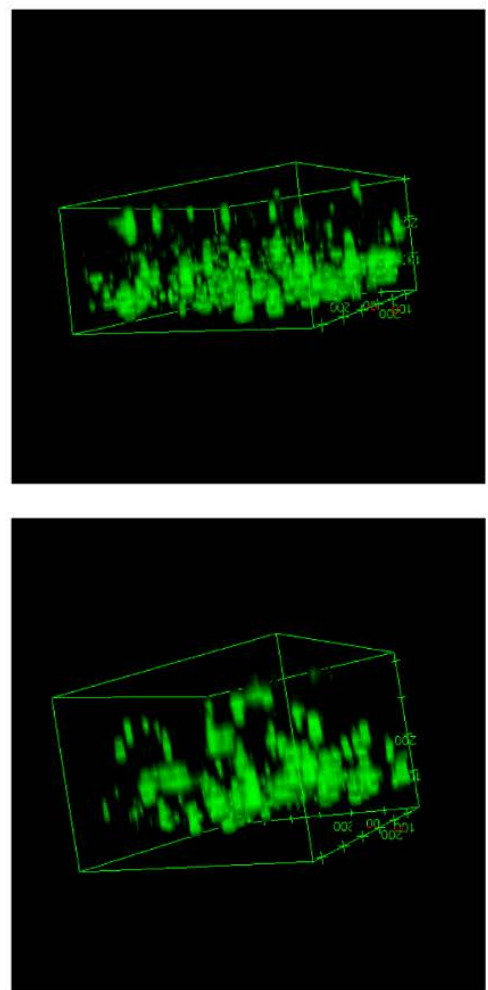

Figure 1S. Cell distribution in Col I/II hydrogels. (A) Maximum intensity projections of Z-stacks. (B-C) Two views of a 3D image from Z-stacks including the (B) bottom and (C) side view of cells seeded at either $1 \times 10^{6}$ or $5 \times 10^{5}$ cells $/ \mathrm{mL}$ and encapsulated in $10 \mu \mathrm{L}$ Col I/II hydrogels. Scale bar $=50 \mu \mathrm{m}$.

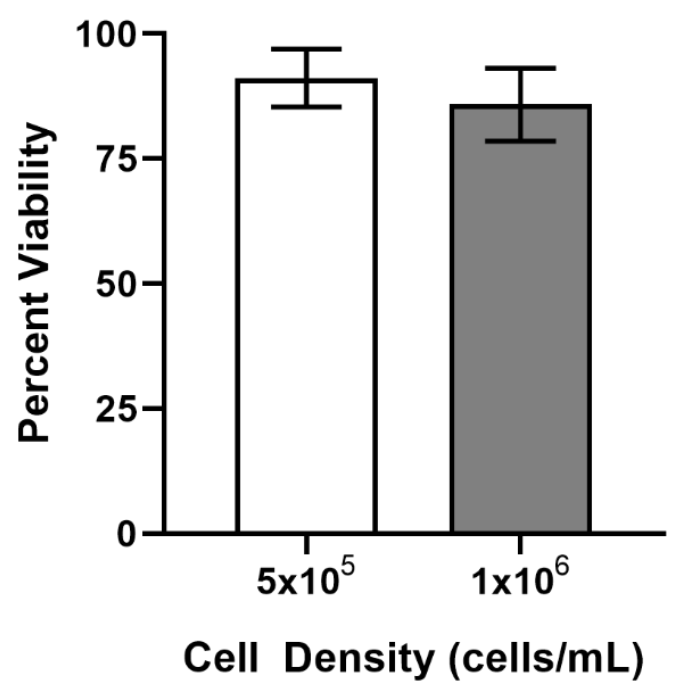

Figure 2S. Live/dead viability assay on mesenchymal stem cells encapsulated at either $1 \times 10^{6}$ or $5 \times 10^{5}$ cells/mL in $10 \mu \mathrm{L}$ Col I/II blend hydrogels for 3 days $(\mathrm{n}=3)$. 


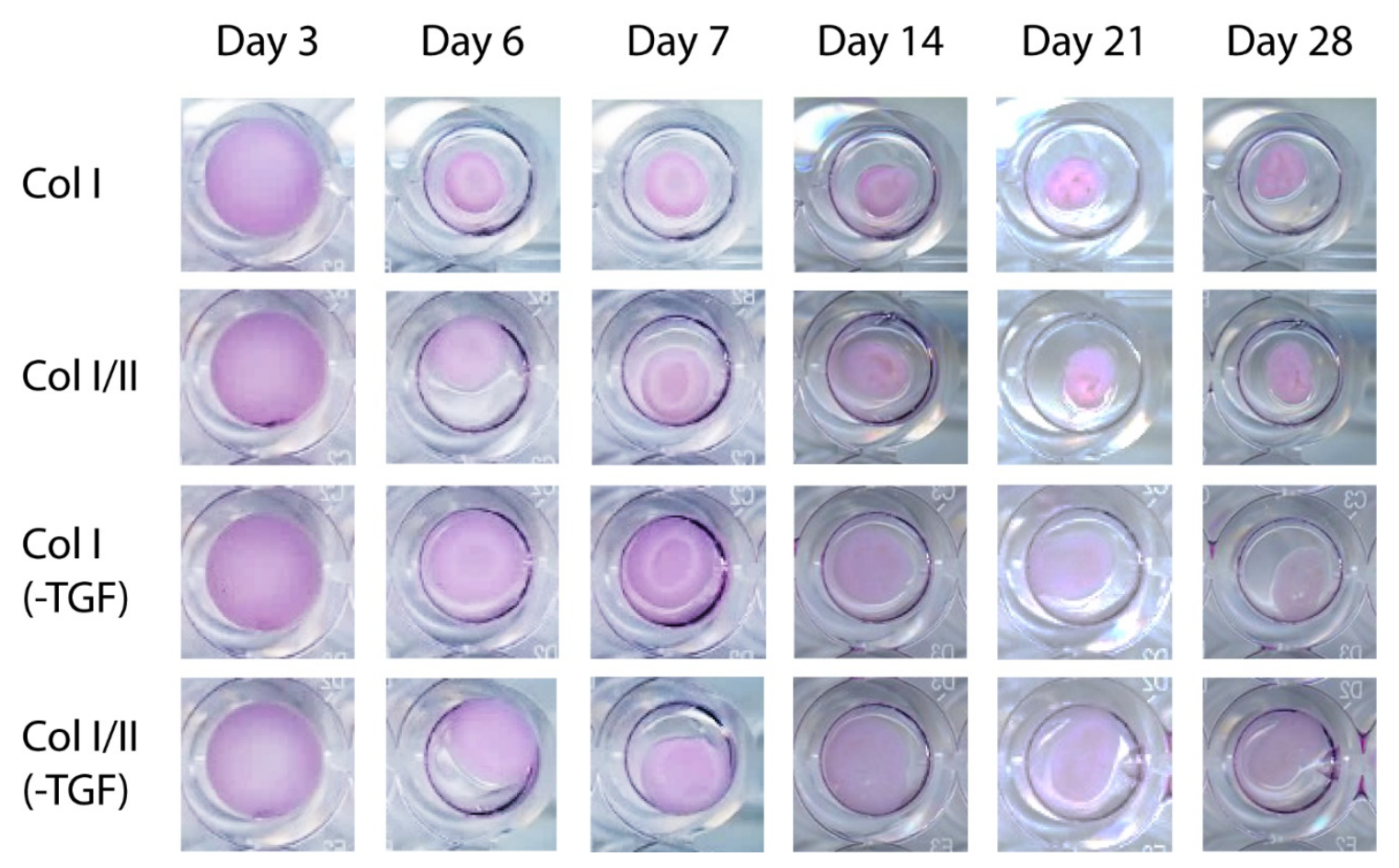

Figure 3S. Pictures of the hydrogels were taken throughout the culture duration when cultured in chondrogenic medium with or without TGF- $\beta 3$, and the surface area of each hydrogel was measured at different time points to investigate contraction. 


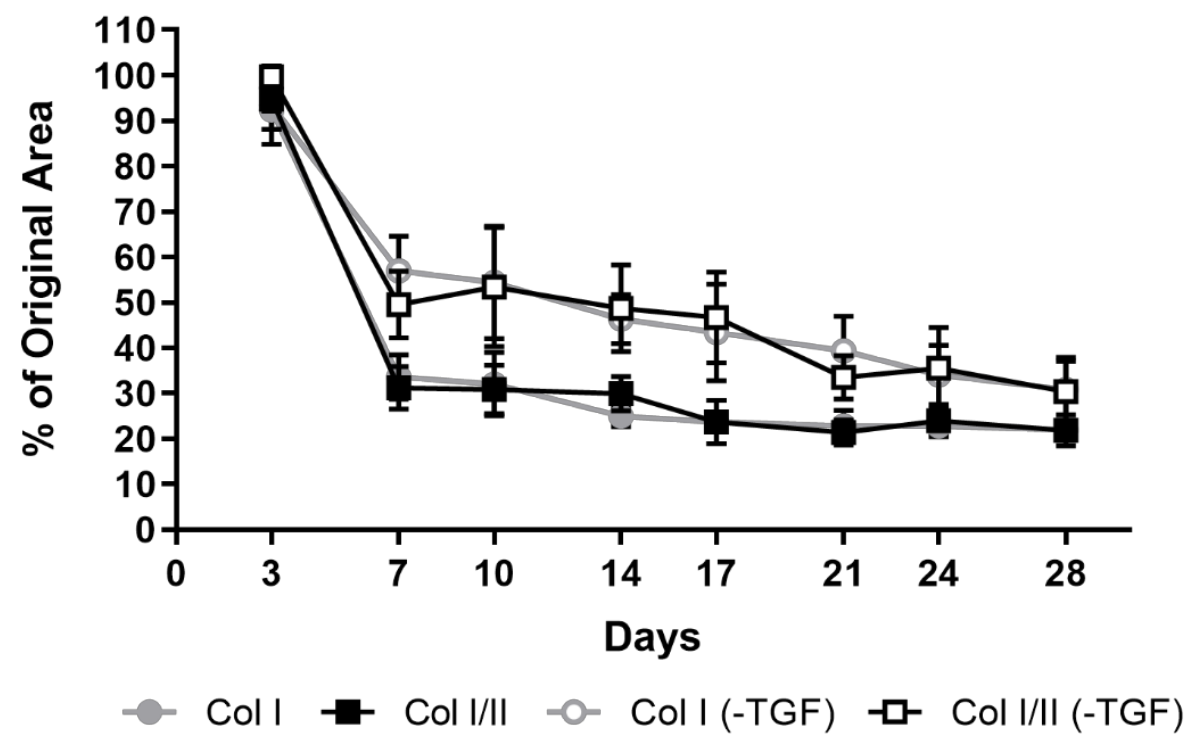

Figure 4S. Comparison of the percentage of the original projected surface area of cell-hydrogel constructs cultured over a 28-day period in chondrogenic medium with or without TGF- $\beta 3$ to examine the contraction of the cell-seeded hydrogels over time. Values are expressed as mean $\pm \mathrm{SD}(n=4)$. At day 3 , a Kruskal-Wallis with Dunn's multiple comparisons test revealed no statistical difference in percentage of the original area between the Col I and Col I/II hydrogels cultured in chondrogenic medium with or without TGF- $\beta 3$. In addition, after 28 days in culture there was no statistical difference in contraction of the hydrogels regardless of medium or hydrogel composition. At all other timepoints, the hydrogels cultured with TGF- $\beta$ contracted more quickly than hydrogels cultured without TGF- $\beta 3$, regardless of hydrogel. 
Table 3S. Averaged glycosaminoglycan normalized to DNA (GAG/DNA), averaged GAG, averaged DNA, and averaged dry weight (DW) values at 14 days of culture.

\begin{tabular}{ccccc}
\hline & & Day 14 & \\
& GAG/DNA & GAG $(\mathrm{ng})$ & DNA $(\mathrm{ng})$ & DW $(\mathrm{mg})$ \\
\hline Col I & $16.2 \pm 6.9$ & $59488.1 \pm 29273.8$ & $4086.4 \pm 2110.4$ & $0.50 \pm 0.08$ \\
Col I/II & $15.6 \pm 1.5$ & $100765.6 \pm 15239.6$ & $6486.2 \pm 992.8$ & $0.62 \pm 0.05$ \\
Pel & $9.8 \pm 2.8$ & $48746.7 \pm 39052.2$ & $5142.4 \pm 3989.2$ & N/A \\
Col I (-TGF) & $4.0 \pm 0.5$ & $35451.2 \pm 5756.1$ & $8178.5 \pm 828.6$ & $0.53 \pm 0.06$ \\
Col I/II (-TGF) & $4.0 \pm 1.0$ & $30402.5 \pm 5484.1$ & $7626.7 \pm 558.2$ & $0.65 \pm 0.12$ \\
Pel (-TGF) & $2.0 \pm 0.8$ & $8489.1 \pm 6356.3$ & $4665.1 \pm 3193.7$ & N/A \\
\hline
\end{tabular}

Table 4S. Averaged glycosaminoglycan normalized to DNA (GAG/DNA), averaged GAG, averaged DNA, and averaged dry weight (DW) values at 28 days of culture.

\begin{tabular}{ccccc}
\hline & & Day 28 & \\
& GAG/DNA & GAG $(\mathrm{ng})$ & DNA $(\mathrm{ng})$ & DW $(\mathrm{mg})$ \\
\hline Col I & $26.8 \pm 7.4$ & $59712.9 \pm 7215.2$ & $2903.8 \pm 867.7$ & $0.28 \pm 0.1$ \\
Col I/II & $43.1 \pm 9.1$ & $78664.0 \pm 14462.5$ & $2183.7 \pm 403.0$ & $0.33 \pm 0.2$ \\
Pel & $22.1 \pm 7.9$ & $38605.6 \pm 8909.1$ & $1900.8 \pm 696.1$ & N/A \\
Col I (-TGF) & $17.6 \pm 3.8$ & $71891.5 \pm 7821.2$ & $4162 \pm 549.9$ & $0.27 \pm 0.1$ \\
Col I/II (-TGF) & $20.0 \pm 5.2$ & $66711.8 \pm 10522.0$ & $3438.9 \pm 589.2$ & $0.23 \pm 0.0$ \\
Pel (-TGF) & $2.4 \pm 1.2$ & $2706.0 \pm 960.4$ & $1223.0 \pm 345.1$ & N/A \\
\hline
\end{tabular}


Medial Condyle - Blank Trochlear Groove - Blank

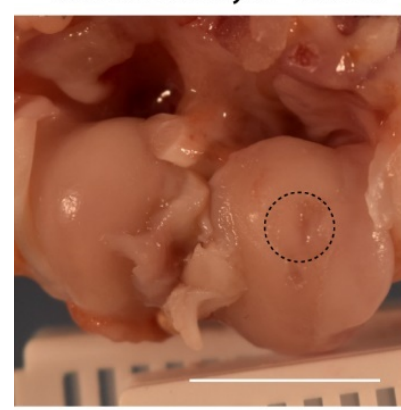

Medial Condyle - Blank

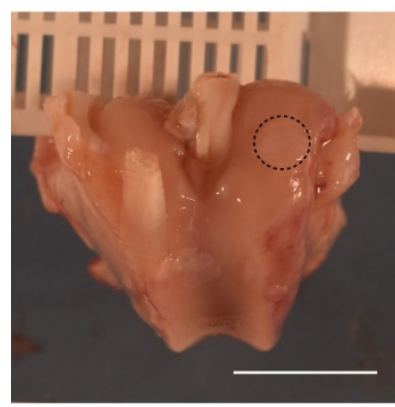

Medial Condyle - Blank

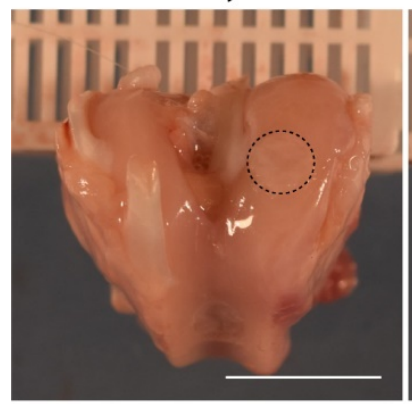

Medial Condyle - Blank

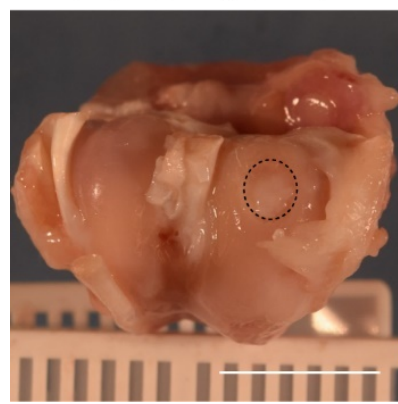

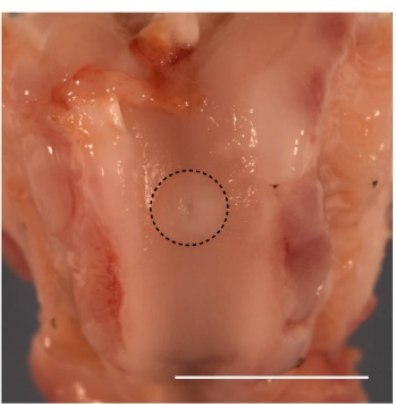

Trochlear Groove - Blank

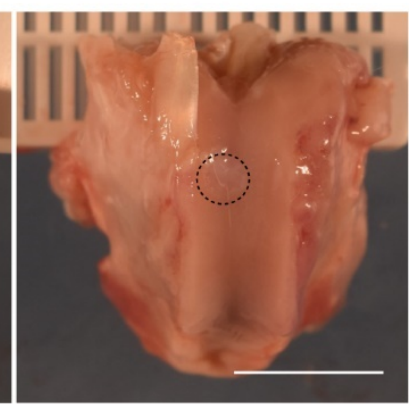

Trochlear Groove - Blank

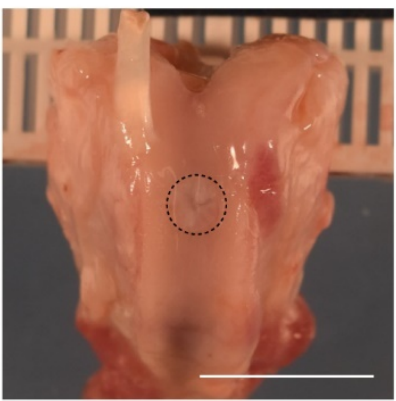

Trochlear Groove - Blank

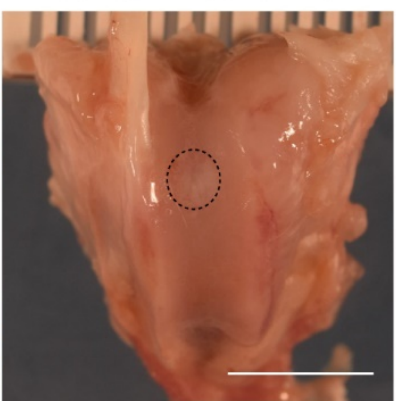

Medial Condyle - Col I/II Trochlear Groove - Col I/II

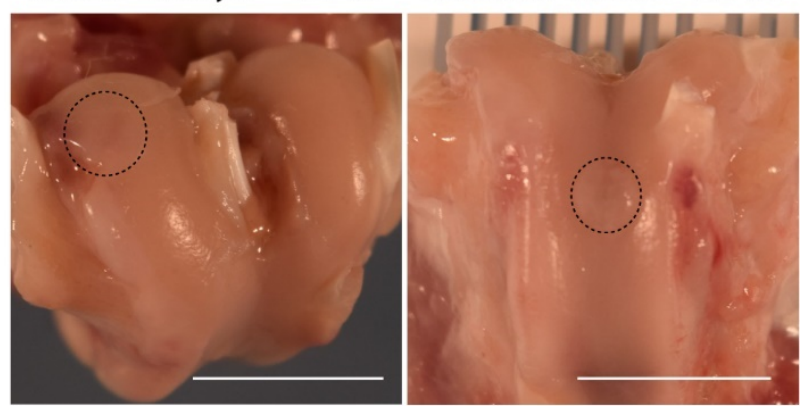

Medial Condyle - Col I/II Trochlear Groove - Col I/II

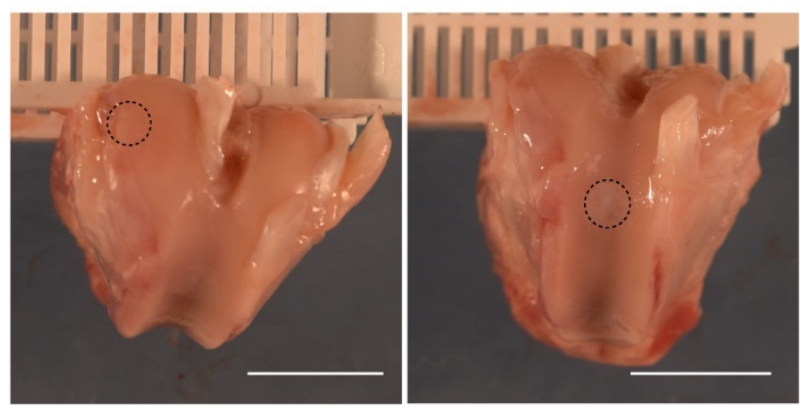

Medial Condyle - Col I/II Trochlear Groove - Col I/II

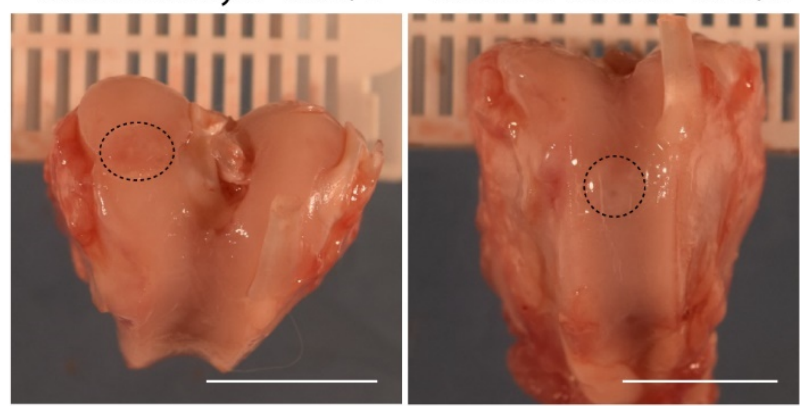

Medial Condyle - Col I/II Trochlear Groove - Col I/II

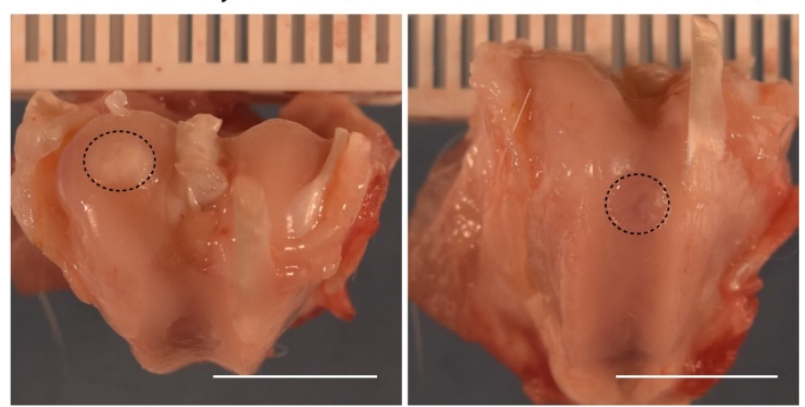

Figure 5S. Macroscopic images of cartilage defects filled with Col I/II hydrogels in the right knee and empty in the left knee of a rabbit. The scale bars represent $1.0 \mathrm{~cm}$ on each image, and repair tissue is surrounded by a dotted line. Macroscopic scoring results are shown in Figure 9S. Each set of four images in a row are from the same rabbit. 


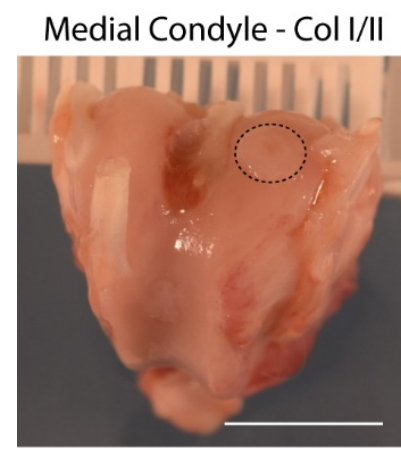

Trochlear Groove - Col I/II
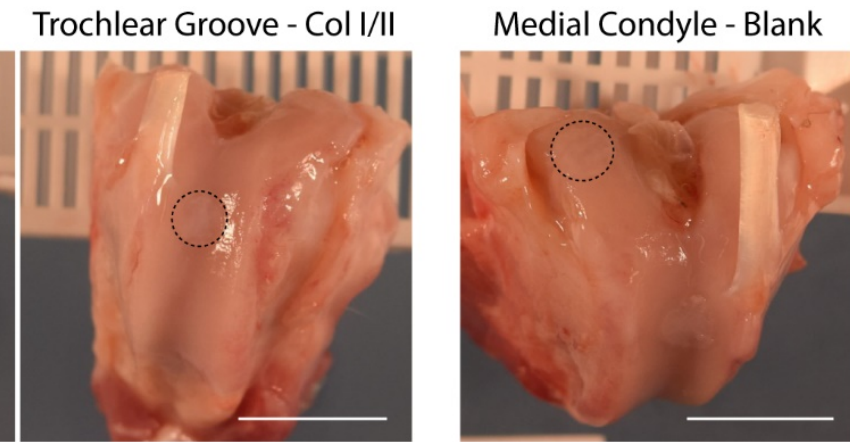

Trochlear Groove - Blank
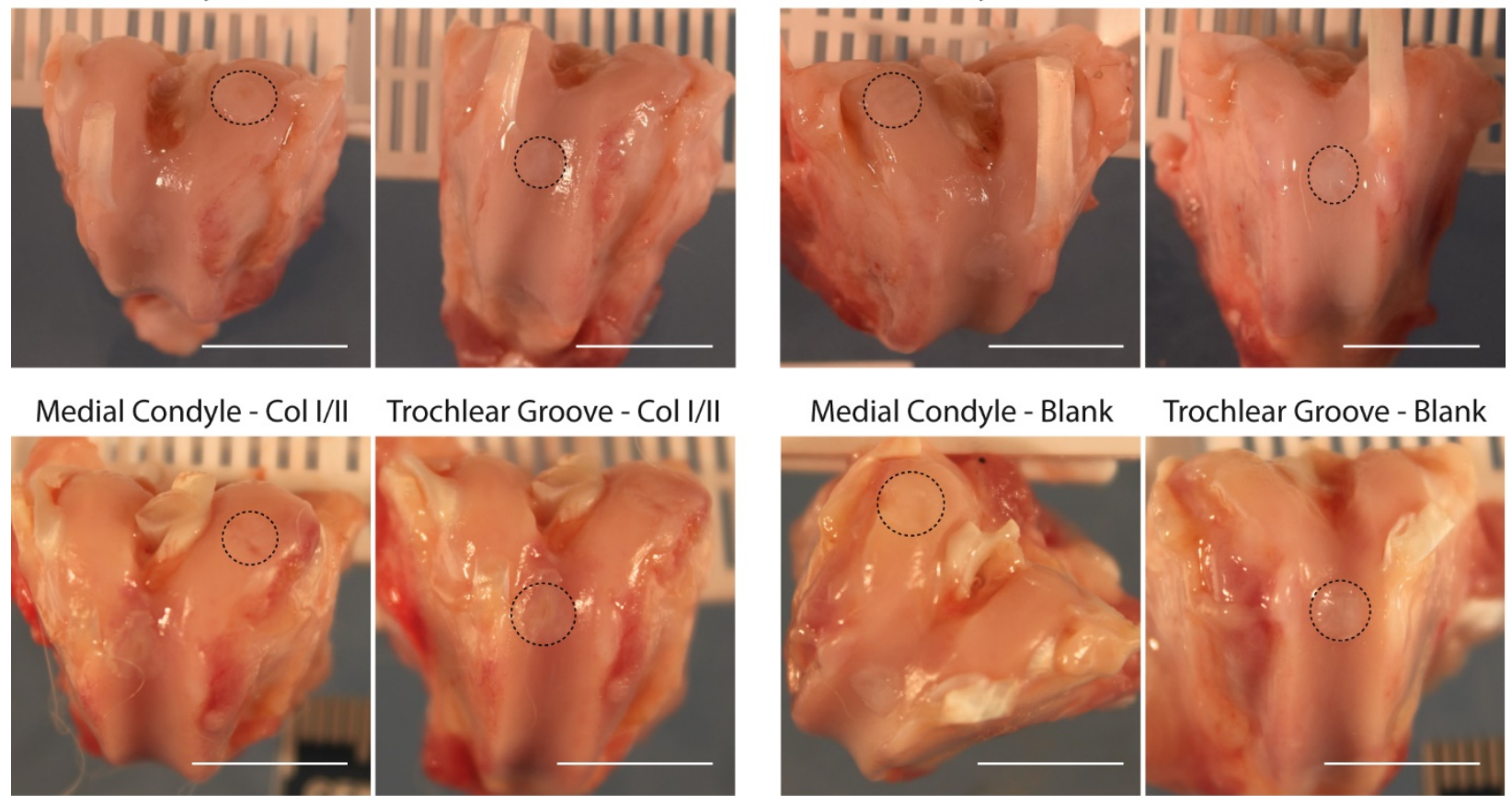

Medial Condyle - Col I/II Trochlear Groove - Col I/II
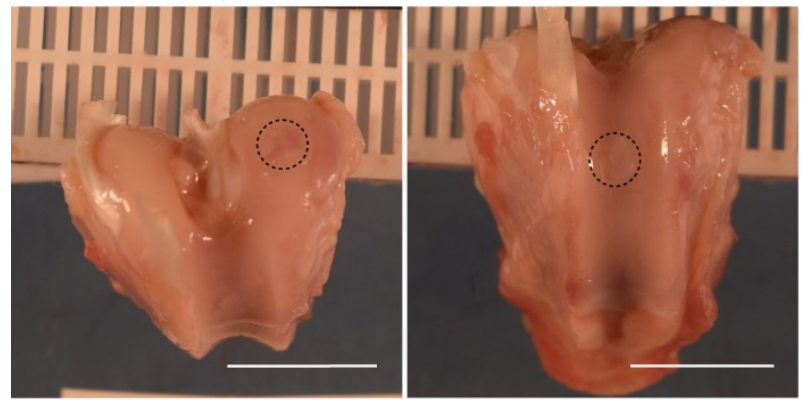

Medial Condyle - Blank

Trochlear Groove - Blank

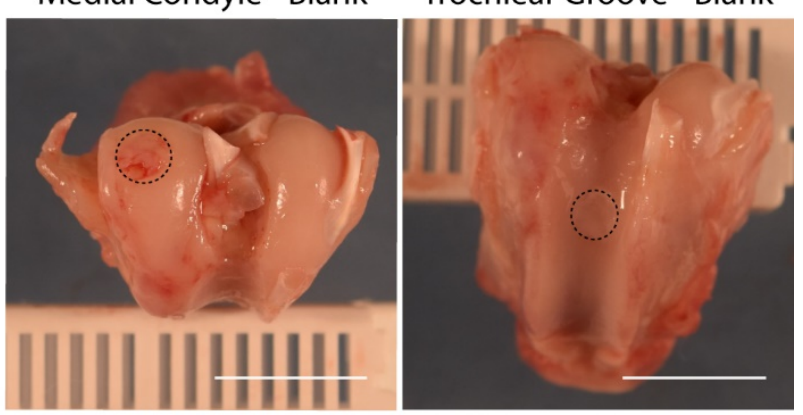

Medial Condyle - Col I/II Trochlear Groove - Col I/II
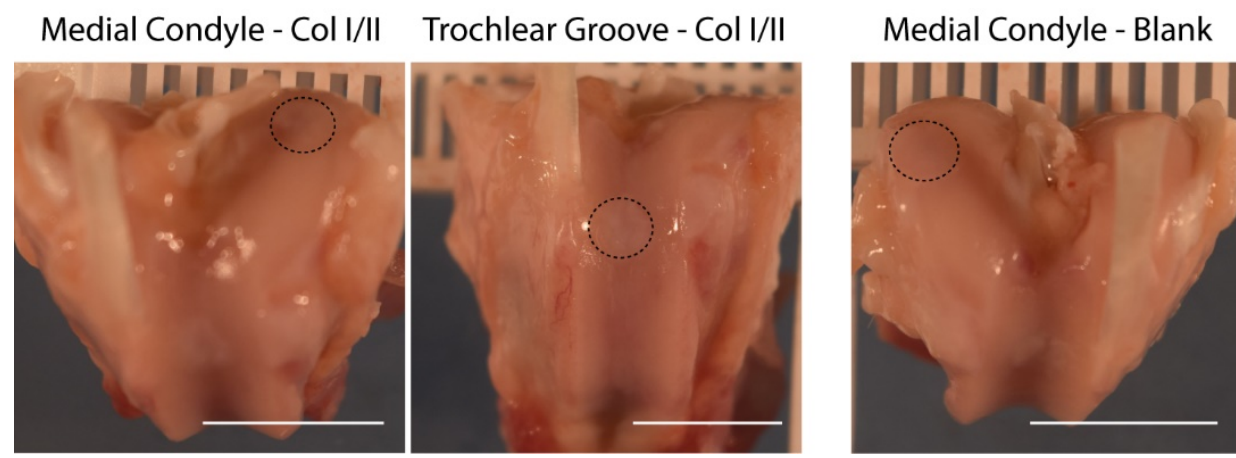

Trochlear Groove - Blank
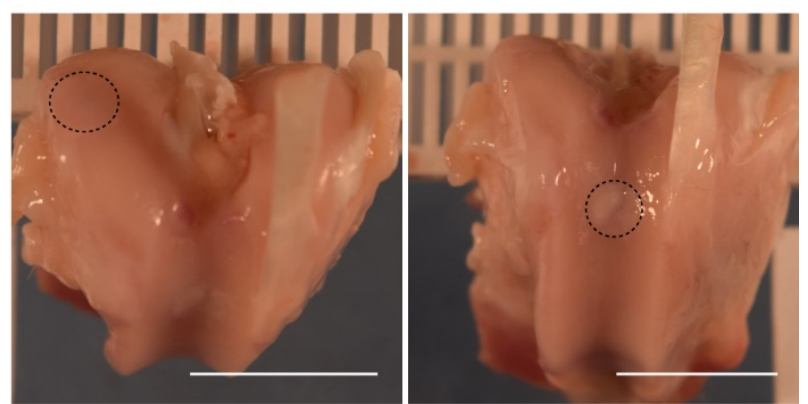

Figure 6S. Macroscopic images of cartilage defects filled with Col I/II hydrogels in the left knee and empty in the right knee of a rabbit. The scale bars represent $1.0 \mathrm{~cm}$ on each image, and repair tissue is surrounded by a dotted line. Macroscopic scoring results are shown in Figure 9S. Each set of four images in a row are from the same rabbit. 

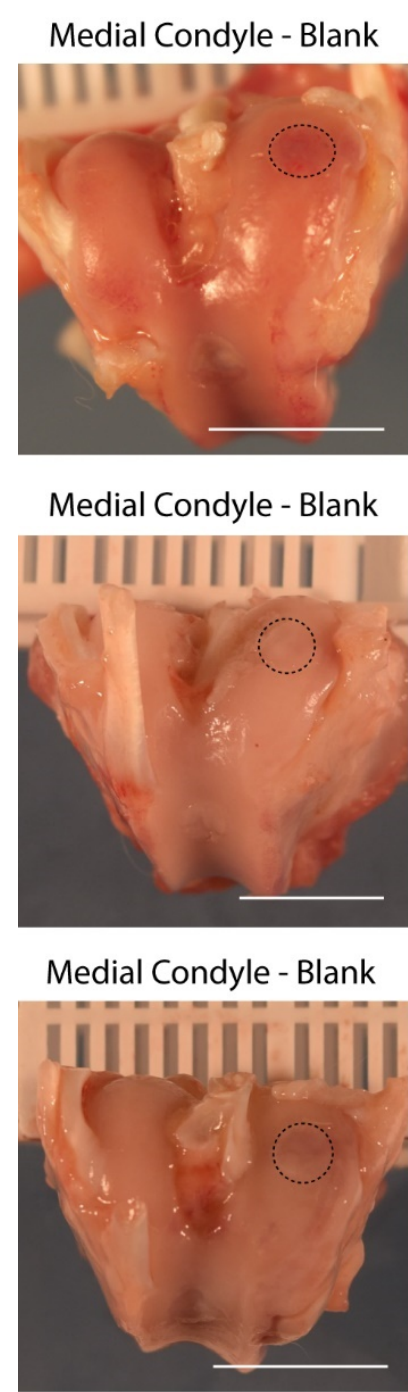

Medial Condyle - Blank

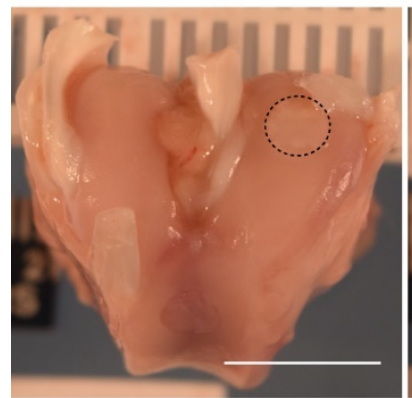

Trochlear Groove - Blank

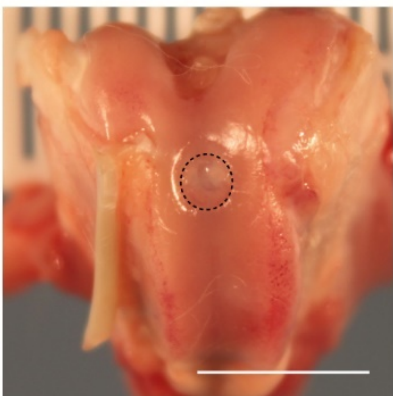

Trochlear Groove - Blank

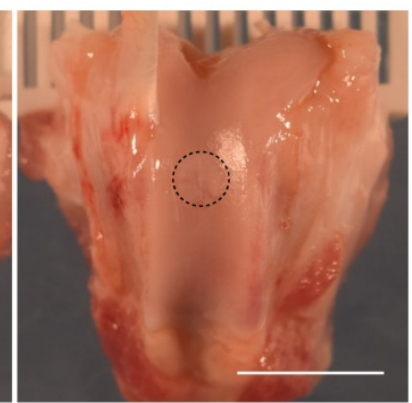

Trochlear Groove - Blank

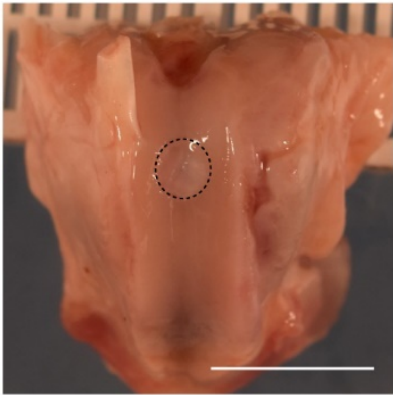

Trochlear Groove - Blank

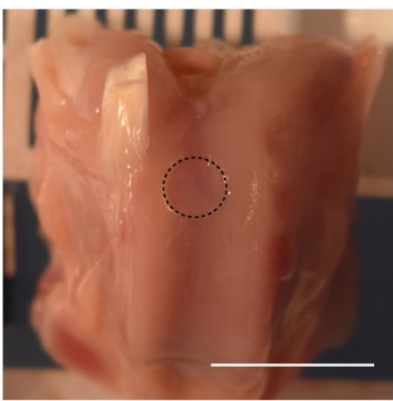

Medial Condyle - Col I Trochlear Groove - Col I

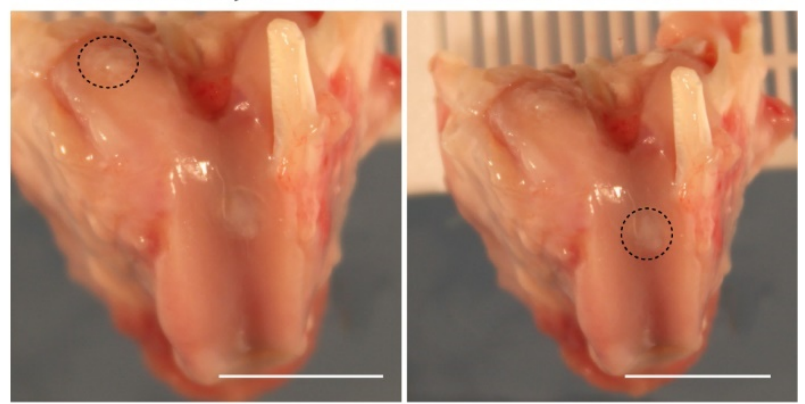

Trochlear Groove - Col I

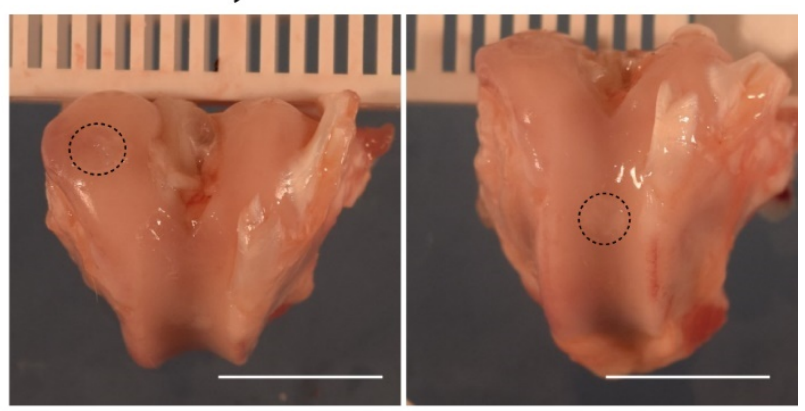

Trochlear Groove - Col I
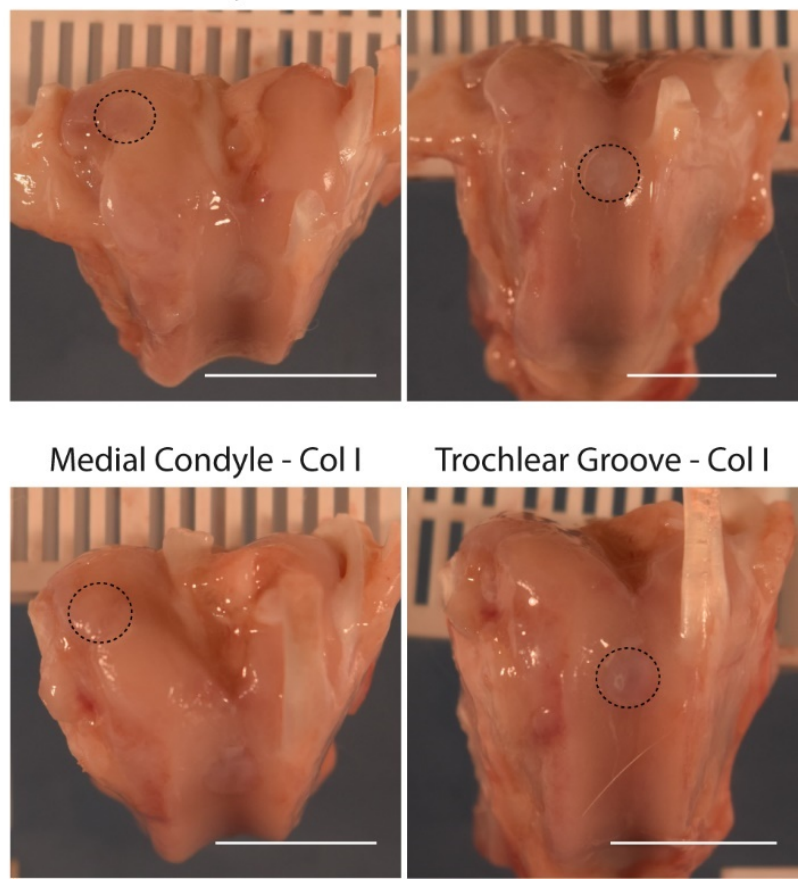

Figure 7S. Macroscopic images of cartilage defects filled with Col I hydrogels in the right knee and empty in the left knee of a rabbit. The scale bars represent $1.0 \mathrm{~cm}$ on each image, and repair tissue is surrounded by a dotted line. Macroscopic scoring results are shown in Figure 9S. Each set of four images in a row are from the same rabbit. 

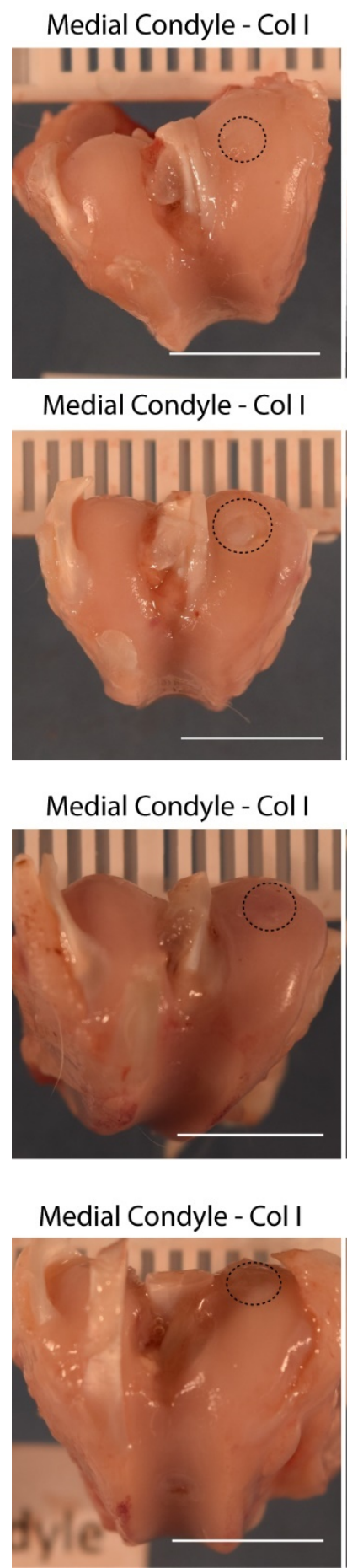

Trochlear Groove - Col I

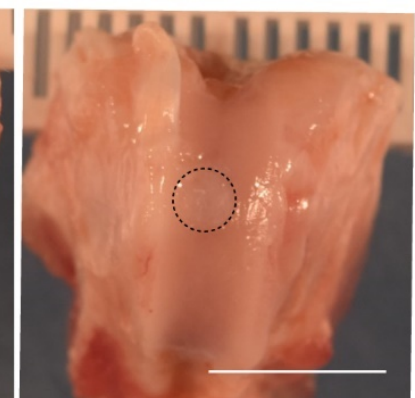

Trochlear Groove - Col I

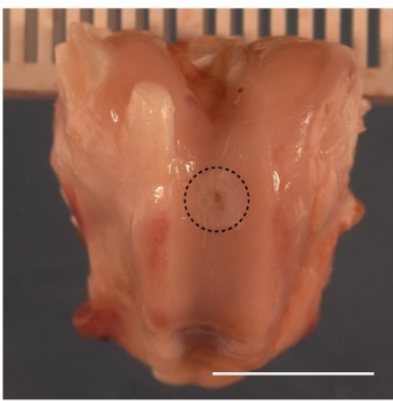

Trochlear Groove - Col I

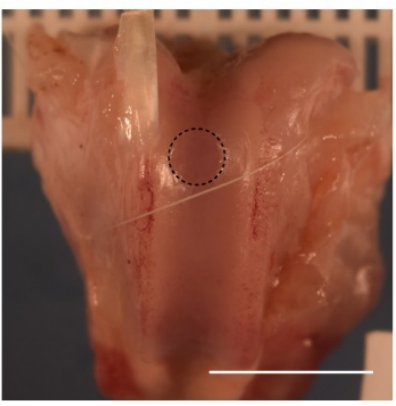

Trochlear Groove - Col I

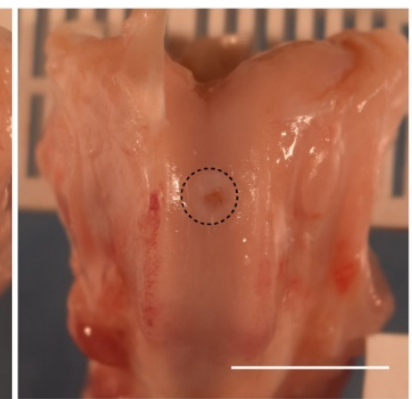

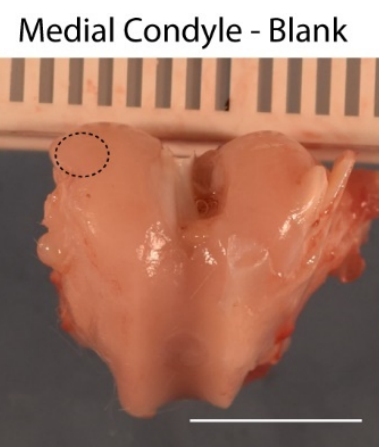

Medial Condyle - Blank

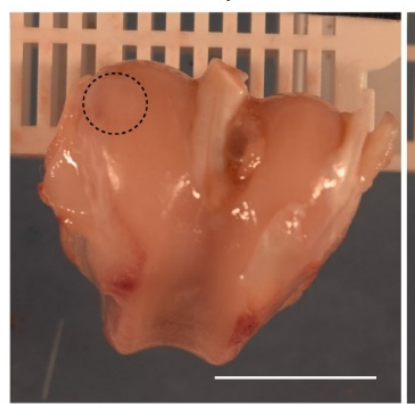

Medial Condyle - Blank

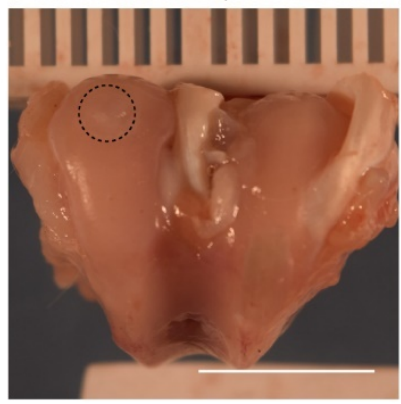

Medial Condyle - Blank Trochlear Groove - Blank

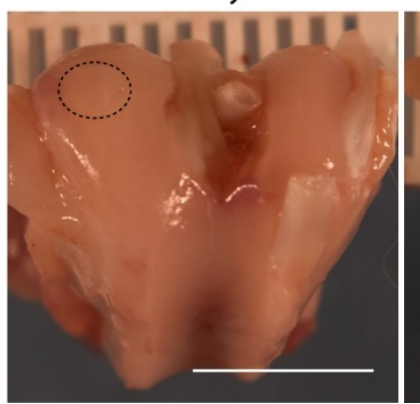

Trochlear Groove - Blank

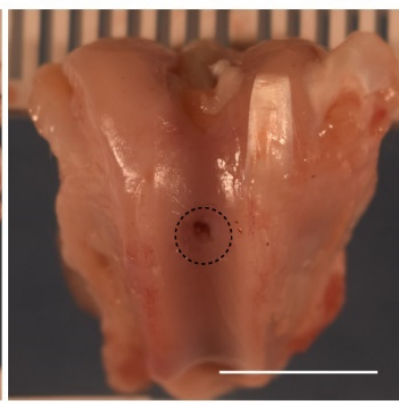

Trochlear Groove - Blank

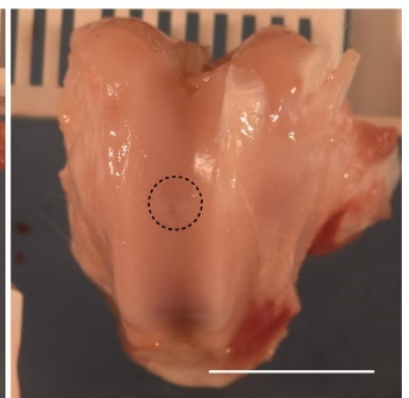

Trochlear Groove - Blank
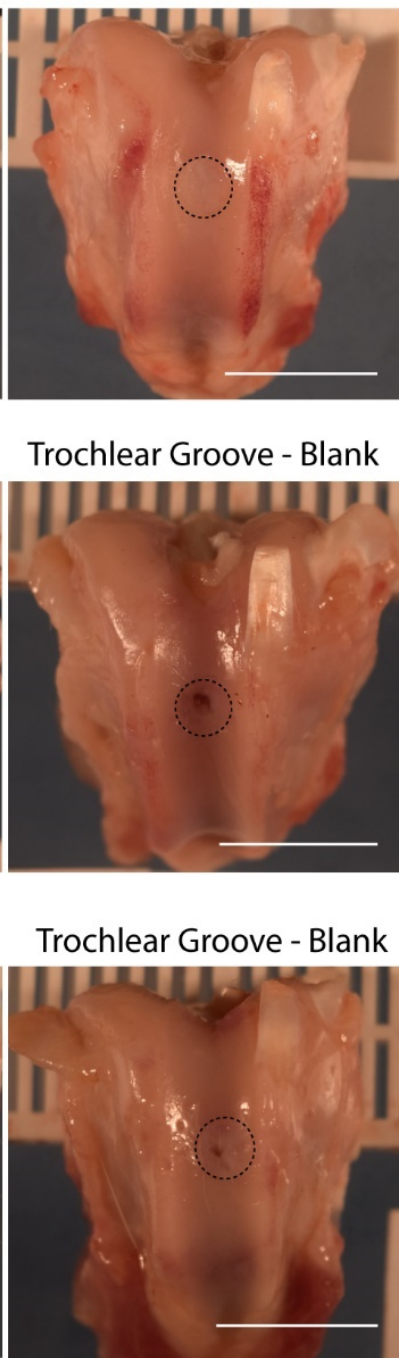

Figure 8S. Macroscopic images of cartilage defects filled with Col I hydrogels in the left knee and empty in the right knee of a rabbit. The scale bars represent $1.0 \mathrm{~cm}$ on each image, and repair tissue is surrounded by a dotted line. Macroscopic scoring results are shown in Figure 9S. Each set of four images in a row are from the same rabbit. 
Table 5S. Macroscopic grading scale adapted from Van den Borne et al ${ }^{1}$.

\begin{tabular}{ll}
\hline & Score \\
\hline Degree of Defect Repair & 4 \\
\hline In level with surrounding cartilage & 3 \\
$75 \%$ repair of defect depth & 2 \\
$50 \%$ repair of defect depth & 1 \\
$25 \%$ repair of defect depth & 0 \\
$0 \%$ repair of defect depth & 4 \\
\hline Integration to Border Zone & 3 \\
\hline Complete integration with surrounding cartilage & \\
Demarcating border $<1$ mm & 2 \\
$3 / 4^{\text {th }}$ of graft integrated, $1 / 4^{\text {th }}$ with a notable boarder $>1$ mm & 1 \\
width & 0 \\
1/2 & \\
width & graft integrated, $1 / 2^{\text {th }}$ with a notable boarder $>1$ mm \\
From no contact to $1 / 4^{\text {th }}$ of graft integrated & 4 \\
\hline Macroscopic Appearance & 3 \\
\hline Intact smooth surface & 2 \\
Fibrillated surface & 1 \\
Small, scattered fissures or cracks & 0 \\
Several small, or few but large fissures & \\
Total degeneration of grafted area &
\end{tabular}
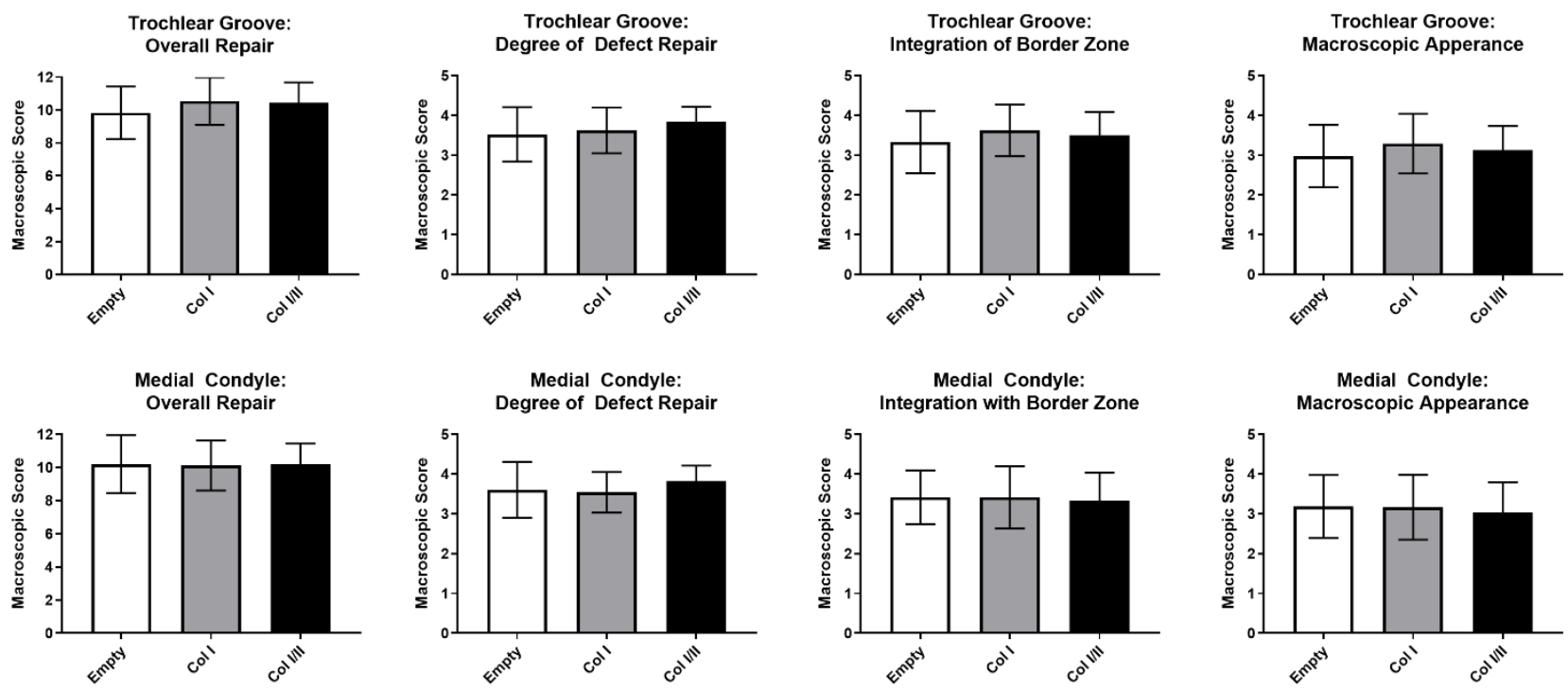

Figure 9S. Macroscopic scoring of repaired cartilage left empty or filled with either a Col I or Col I/II hydrogel. Kruskal-Wallis one-way ANOVAs and Dunn's post hoc tests were performed $(p<0.05)$ for macroscopic scoring analysis. Three blinded observers scored the defects where $n=8$ defects were scored for the Col I/II and Col I defect and n=16 defects were scored for the empty defects. 
A

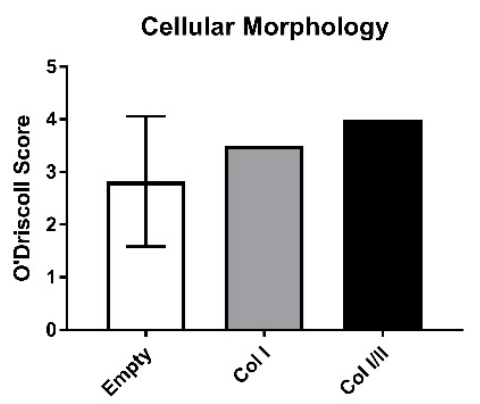

D

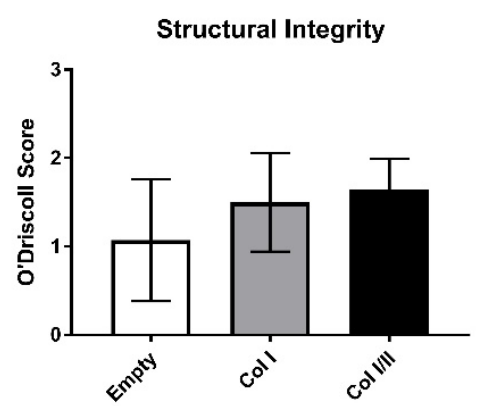

G

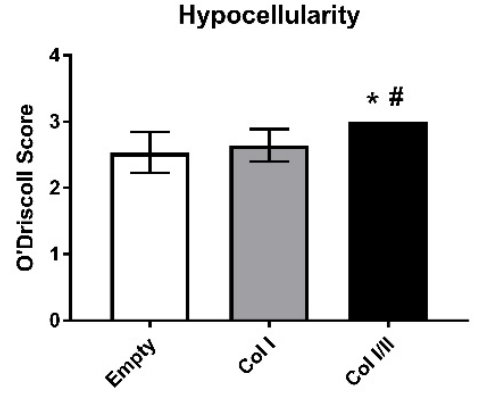

B

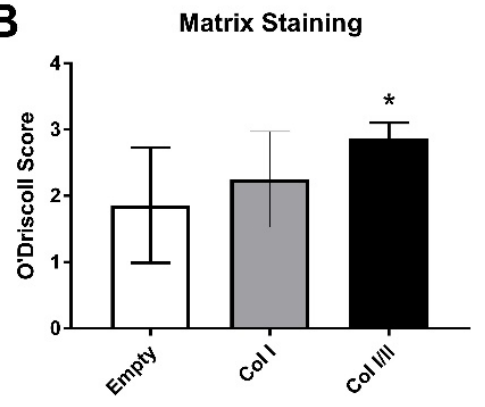

E

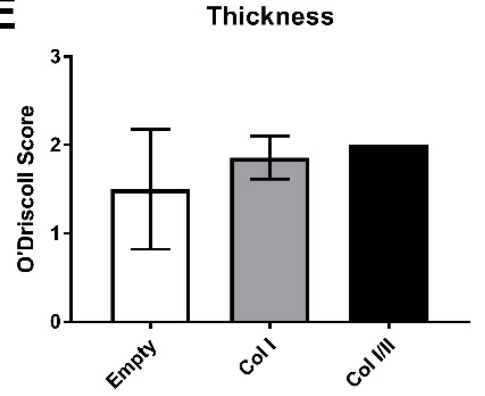

H

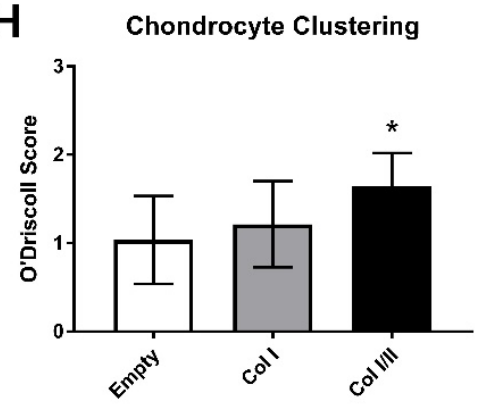

C Surface Regularity

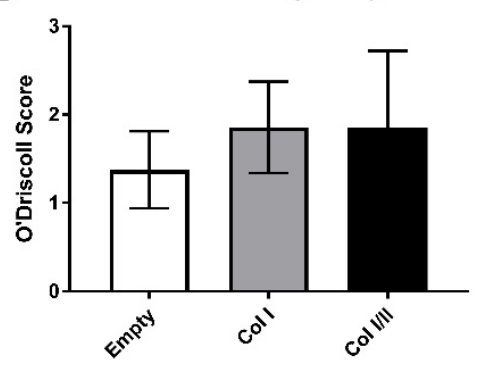

F Bonding to Adjacent Cartilage

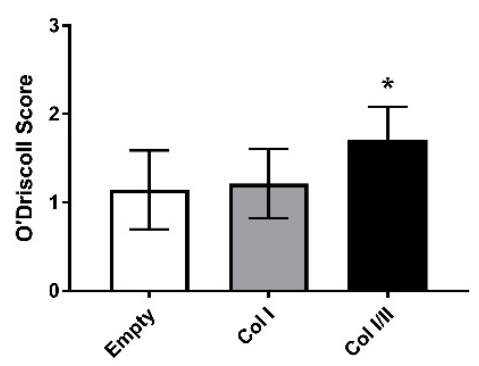

I

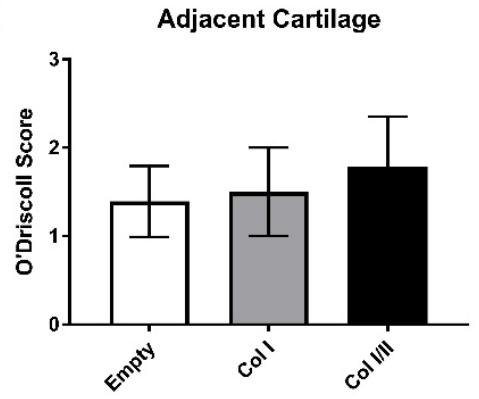

Figure 10S. Histological evaluation of each O'Driscoll scoring category to assess cartilage repair within defects in the trochlear groove. One-way ANOVAs and Tukey's post hoc tests were performed $(\mathrm{p}<0.05)$ for histology scoring analysis. * indicates statistical difference from the empty defect, and \# indicates statistical difference from the Col I hydrogel. 
A

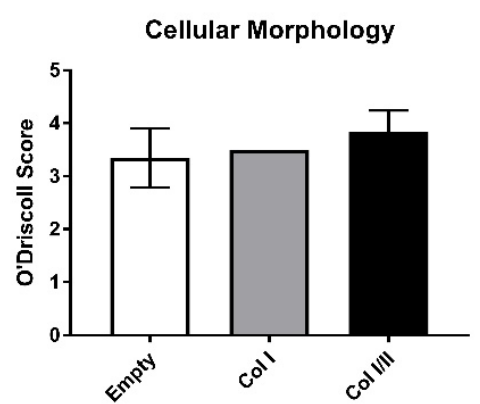

D

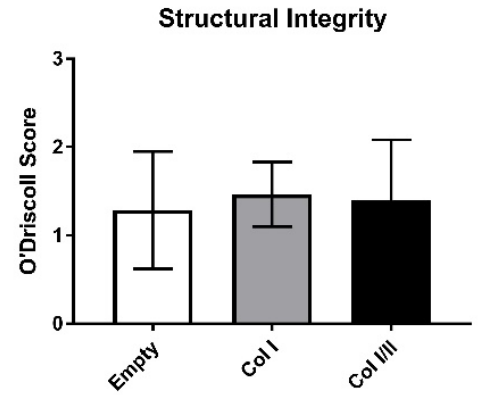

G

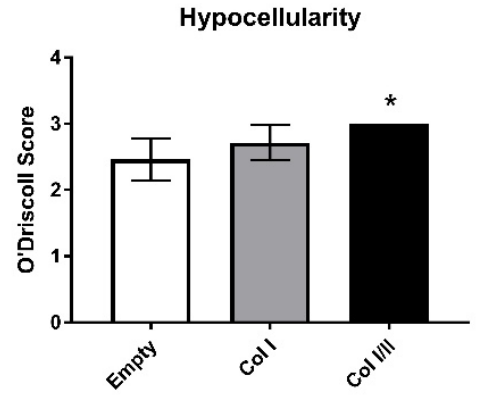

B

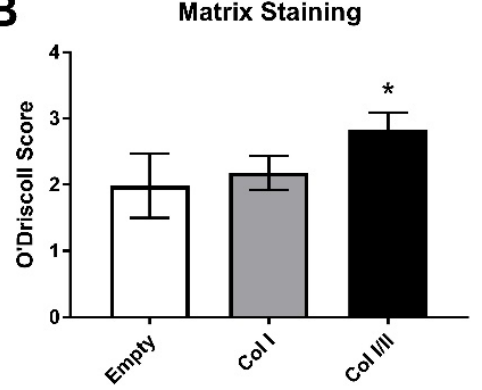

E

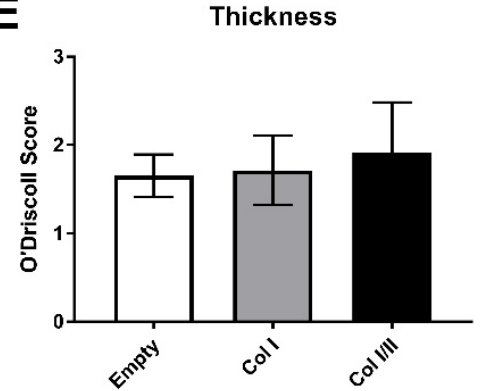

H

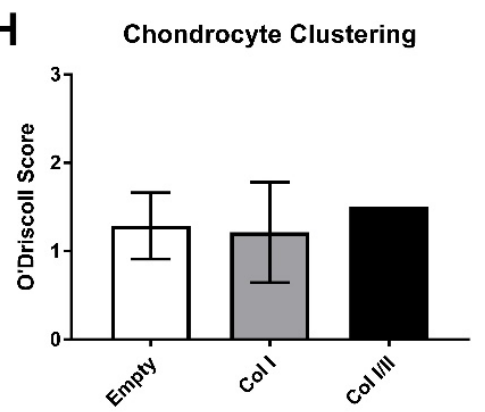

C Surface Regularity

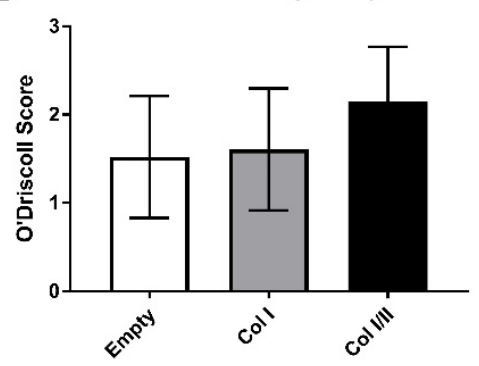

F Bonding to Adjacent Cartilage

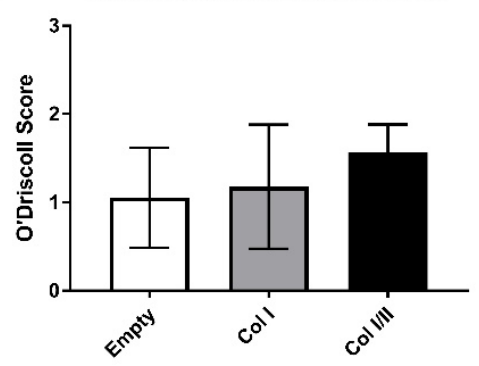

I

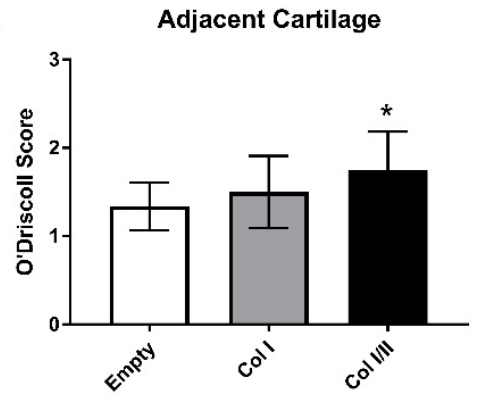

Figure 11S. Histological evaluation of each O'Driscoll scoring category to assess cartilage repair within defects in the medial condyle. One-way ANOVAs and Tukey's post hoc tests were performed $(\mathrm{p}<0.05)$ for histology scoring analysis. * indicates statistical difference from the empty defect, and \# indicates statistical difference from the Col I hydrogel. 
Table 6S. Polymerization conditions to create a hydrogel with mechanical properties that match those of the Col I/II blend hydrogel.

\begin{tabular}{cccc}
\hline Name & $\begin{array}{c}\text { Pre- } \\
\text { polymerization } \\
\text { Ratio of Col I:II }\end{array}$ & $\begin{array}{c}\text { Pre- } \\
\text { polymerization } \\
\text { concentration } \\
(\mathrm{mg} / \mathrm{mL})\end{array}$ & $\begin{array}{c}\text { Post- } \\
\text { polymerization } \\
\text { concentration } \\
(\mathrm{mg} / \mathrm{mL})\end{array}$ \\
\hline $\begin{array}{c}\text { Collagen Type I/II } \\
\text { (Col I/II) } \\
\text { Mechanically } \\
\text { Matched Col I } \\
\text { (MM) }\end{array}$ & $3: 1$ & 4 & $3.00 \pm 0.05$ \\
$\begin{array}{c}\text { Collagen Type I } \\
\text { (Col I) }\end{array}$ & $1: 0$ & 3.2 & $2.96 \pm 0.01$ \\
\hline
\end{tabular}
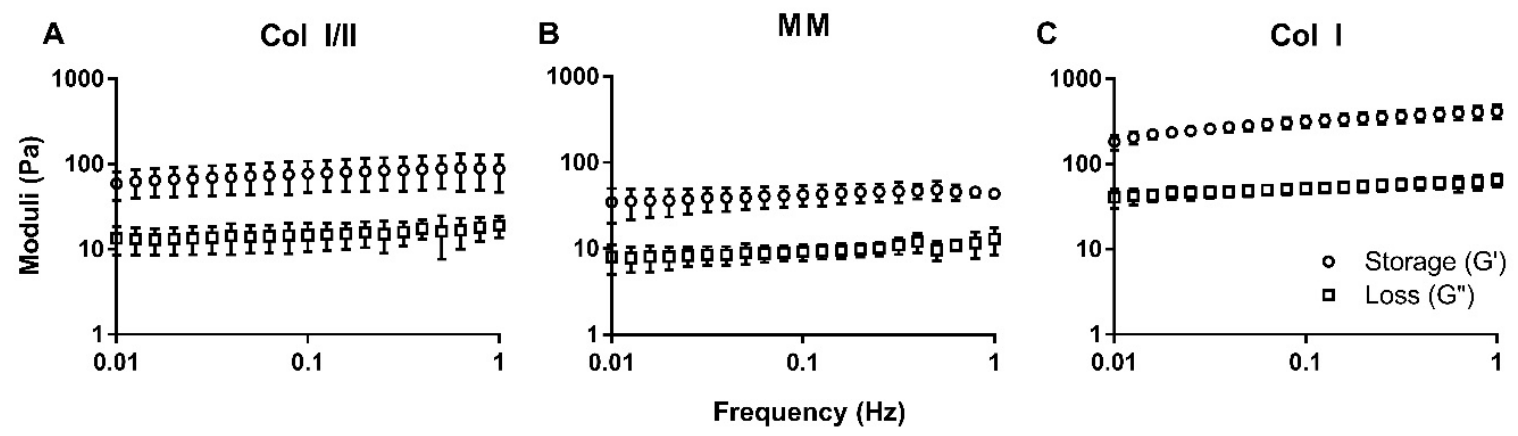

Figure 12S. Frequency sweeps confirmed that the mechanical properties were statistically similar between the Col I/II and the Col I MM hydrogels $(n=4)$. 

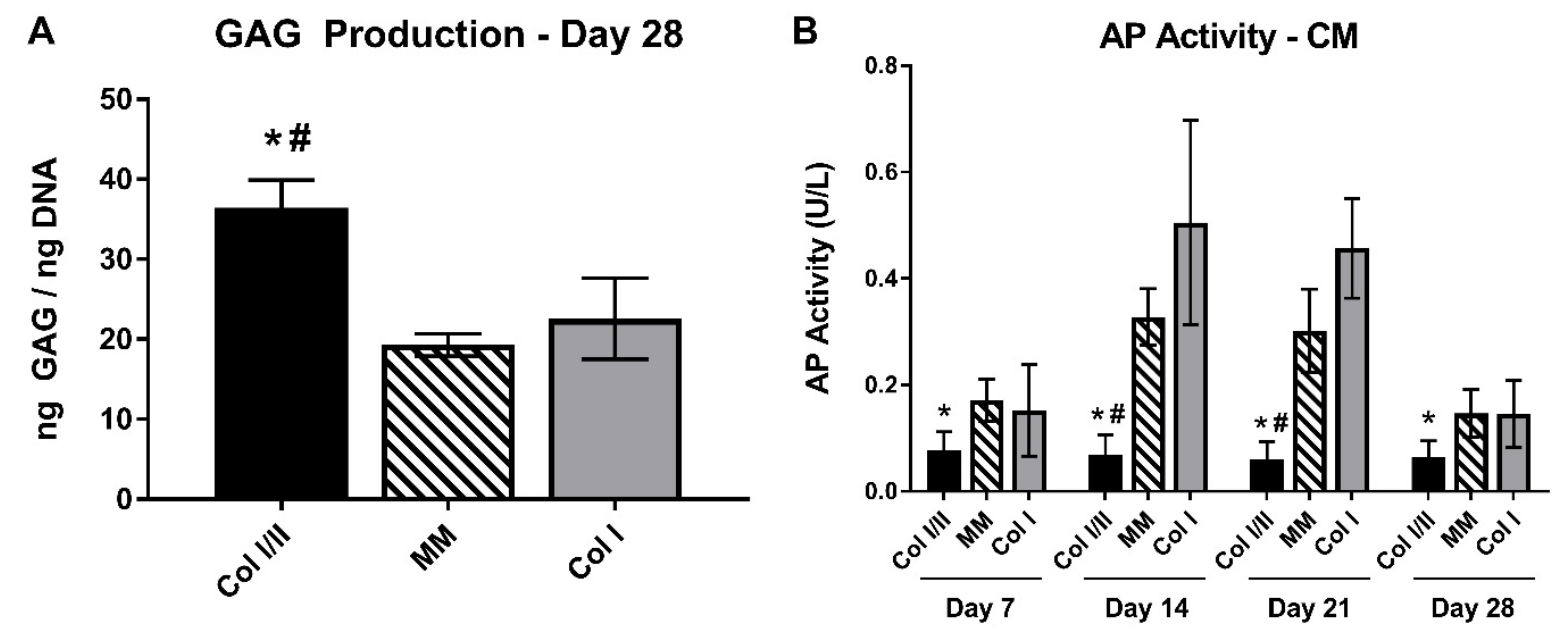

Figure 13S. The addition of collagen type II promoted GAG synthesis and did not increase alkaline phosphatase (AP) activity, and these effects were independent of mechanical properties. (A) GAG/DNA ratio of the cell-hydrogel constructs after a 4-week culture period. Values are expressed as mean \pm SD (n $=4)$. An ANOVA and Tukey's post hoc test were performed $(\mathrm{p}<0.05)$ * indicates statistical difference from the MM sample, and \# indicates statistical difference from the Col I hydrogel. (B) AP activity of rabbit MSCs over time $(n=4)$. An ANOVA and Tukey's post hoc test were performed $(p<0.05) .{ }^{*}$ indicates statistical difference from the MM sample within a time point, and \# indicates statistical difference from the Col I hydrogel within a time point. 


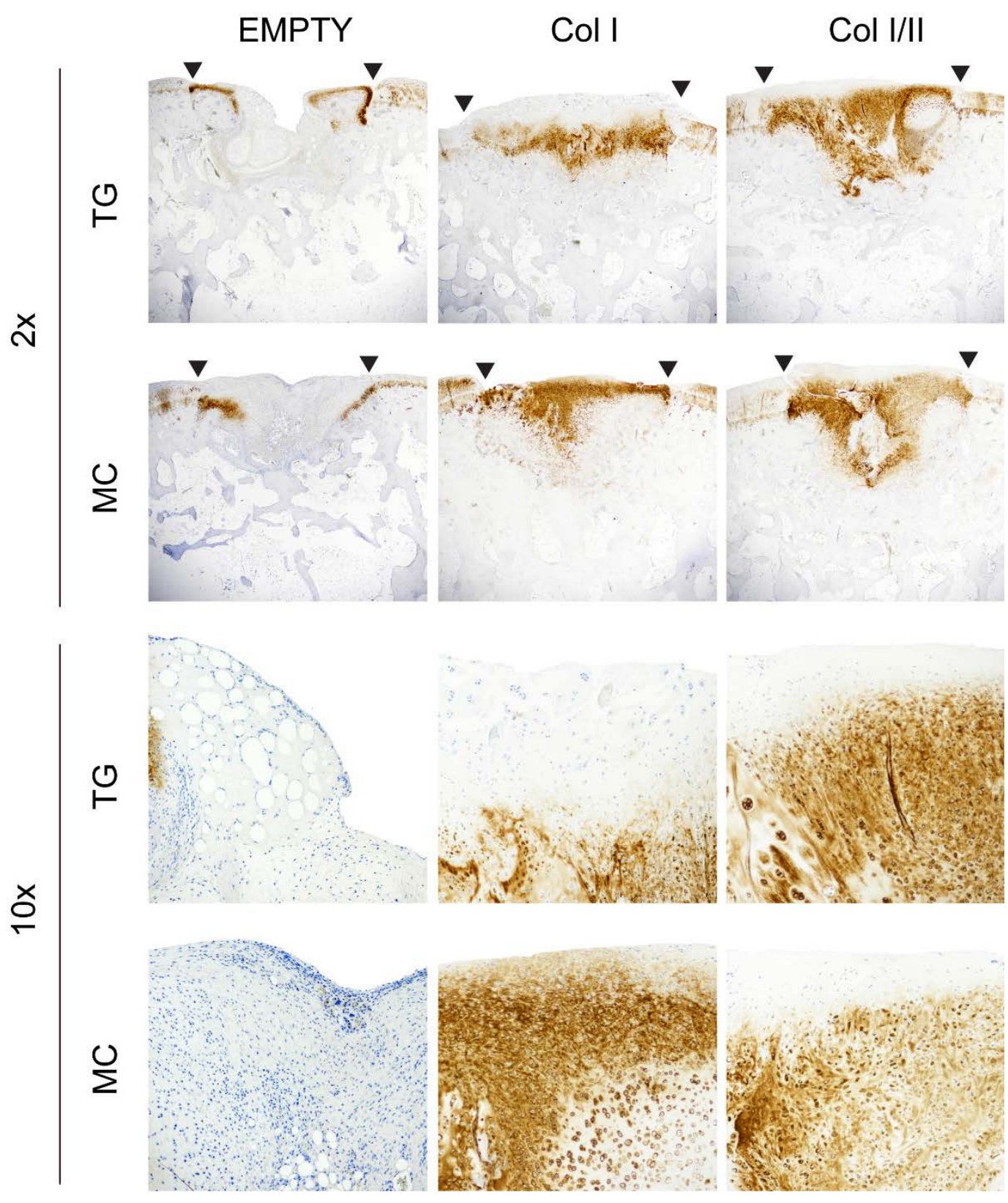

Figure 14S. More collagen type II was labeled in defects that were filled with Col I/II hydrogels compared to defects that were filled with Col I hydrogels or left empty. Collagen type II immunohistochemistry of repaired cartilage left blank or filled with either a Col I or Col I/II hydrogel in the trochlear groove (TG) or the medial condyle (MC) at two different magnifications (2x and 10x) and imaged using a microscope.

Immunohistochemical labeling of collagen type II revealed that collagen type II was present in all defects filled with hydrogel constructs. Brown labeling of collagen type II was present throughout the defect but was most intense adjacent to the bone compared to the tissue surface. This intensity differential was especially noticeable in defects with Col I scaffolds in the TG. The empty defects also contained some staining for collagen type II, but staining was localized to areas adjacent to surrounding tissue. Areas further away from surrounding tissue were void of collagen type II staining. It is difficult to interpret these results since collagen type II was added prior to polymerization in only one treatment group, and surgeries were staggered due to time constraints placed by the surgical team of veterinarians resulting in different amounts of time in fixatives and potential differences in antigen retrieval. Therefore, the collagen type II stained slides were not utilized in the semi-quantitative scoring. 


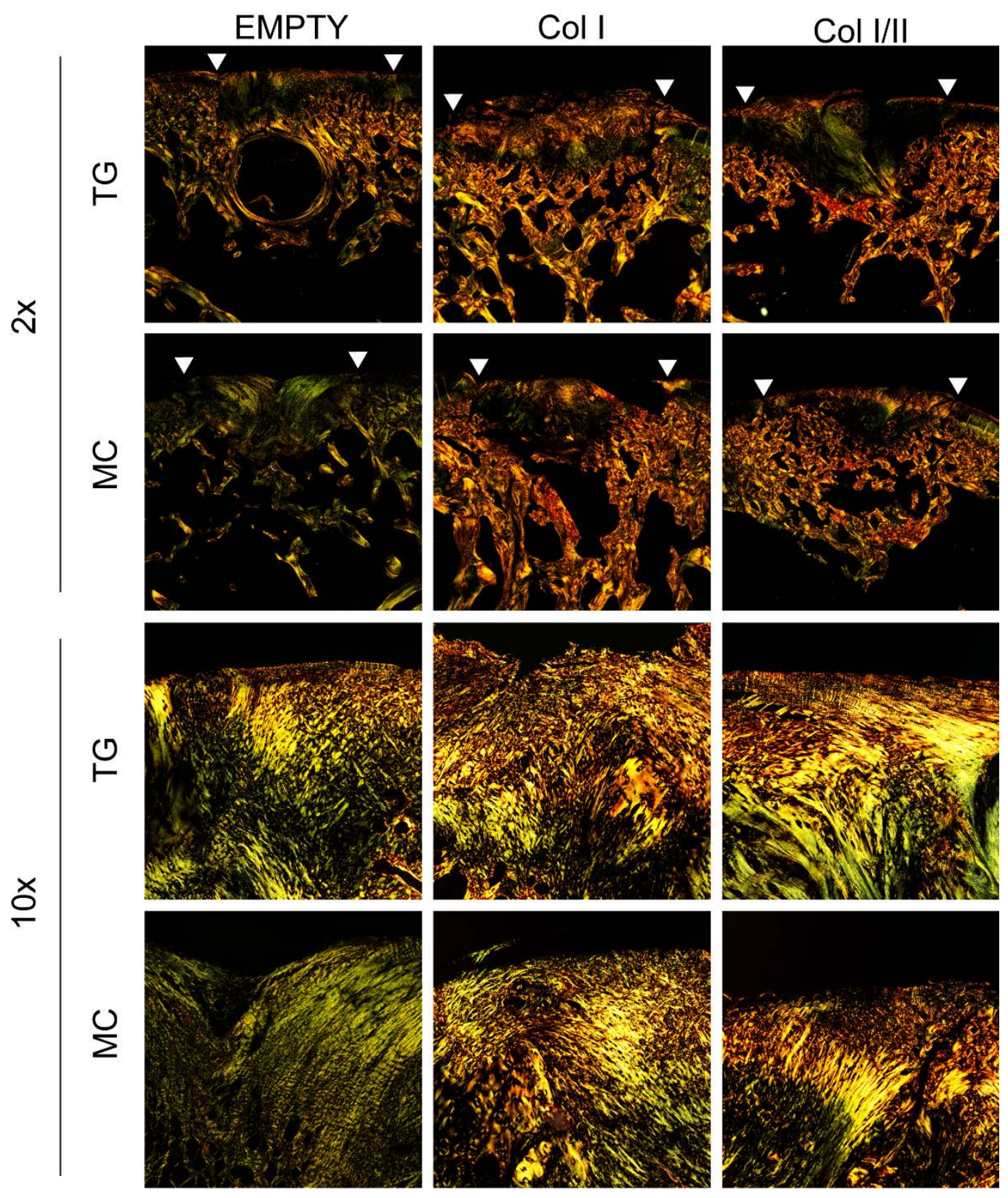

Figure 15S. Picrosirius red staining of repaired cartilage left empty or filled with either a Col I or Col I/II hydrogel. Cartilage repair in two different locations, the trochlear groove (TG) and the medial condyle (MC), was analyzed at 2x and 10x. Picrosirius red was used to stain sections and imaged using polarized light microscopy to analyze collagen organization. Although slight differences between treatments could be distinguished, little to no collagen fiber organization, as seen in native tissue, was observed. 


\section{REFERENCES}

(1) van den Borne, M. P. J.; Raijmakers, N. J. H.; Vanlauwe, J.; Victor, J.; de Jong, S. N.; Bellemans, J.; Saris, D. B. F. International Cartilage Repair Society (ICRS) and Oswestry Macroscopic Cartilage Evaluation Scores Validated for Use in Autologous Chondrocyte Implantation (ACI) and Microfracture. Osteoarthr. Cartil. 2007, 15 (12), 1397-1402. https://doi.org/10.1016/j.joca.2007.05.005.

(2) Douglas, T.; Heinemann, S.; Mietrach, C.; Hempel, U.; Bierbaum, S.; Scharnweber, D.; Worch, H. Interactions of Collagen Types I and II with Chondroitin Sulfates A-C and Their Effect on Osteoblast Adhesion. Biomacromolecules 2007, 8 (4), 1085-1092. https://doi.org/10.1021/bm0609644.

(3) Liao, J.; Guo, X.; Grande-Allen, K. J.; Kasper, F. K.; Mikos, A. G. Bioactive Polymer/Extracellular Matrix Scaffolds Fabricated with a Flow Perfusion Bioreactor for Cartilage Tissue Engineering. Biomaterials 2010, 31 (34), 8911-8920. https://doi.org/10.1016/j.biomaterials.2010.07.110.

(4) Yuan, L.; Li, B.; Yang, J.; Ni, Y.; Teng, Y.; Guo, L.; Fan, H.; Fan, Y.; Zhang, X. Effects of Composition and Mechanical Property of Injectable Collagen I/II Composite Hydrogels on Chondrocyte Behaviors. Tissue Eng. Part A 2016, 22 (11-12), 899-906. https://doi.org/10.1089/ten.tea.2015.0513.

(5) Zheng, L.; Sun, J.; Chen, X.; Wang, G.; Jiang, B.; Fan, H.; Zhang, X. In Vivo Cartilage Engineering with Collagen Hydrogel and Allogenous Chondrocytes after Diffusion Chamber Implantation in Immunocompetent Host. Tissue Eng. Part A 2009, 15 (8), 2145-2153. https://doi.org/10.1089/ten.tea.2008.0268.

(6) Chen, C. H.; Kuo, C. Y.; Wang, Y. J.; Chen, J. P. Dual Function of Glucosamine in Gelatin/Hyaluronic Acid Cryogel to Modulate Scaffold Mechanical Properties and to Maintain Chondrogenic Phenotype for Cartilage Tissue Engineering. Int. J. Mol. Sci. 2016, 17 (11), 1957. https://doi.org/10.3390/ijms17111957.

(7) Zhang, L.; Yuan, T.; Guo, L.; Zhang, X. An in Vitro Study of Collagen Hydrogel to Induce the Chondrogenic Differentiation of Mesenchymal Stem Cells. J. Biomed. Mater. Res. - Part A 2012, 100 A (10), 2717-2725. https://doi.org/10.1002/jbm.a.34194.

(8) Morigele, M.; Shao, Z.; Zhang, Z.; Kaige, M.; Zhang, Y.; Qiang, W.; Yang, S. TGF- $\beta 1$ Induces a Nucleus Pulposus-like Phenotype in Notch 1 Knockdown Rabbit Bone Marrow Mesenchymal Stem Cells. Cell Biol. Int. 2013, 37 (8), 820-825. https://doi.org/10.1002/cbin.10109.

(9) O’Driscoll, S. W.; Keeley, F. W.; Salter, R. B. Durability of Regenerated Articular Cartilage Produced by Free Autogenous Periosteal Grafts in Major Full-Thickness Defects in Joint Surfaces under the Influence of Continuous Passive Motion. A Follow-up Report at One Year. J. Bone Jt. Surgery. 1988, 70 (4), 595-606. https://doi.org/10.2106/00004623-198870040-00017. 